(C2017, Elsevier. Licensed under the Creative Commons Attribution-NonCommercialNoDerivatives 4.0 International http://creativecommons.org/about/downloads 


\title{
Frequency domain subpixel registration using HOG Phase Correlation
}

\author{
Vasileios Argyriou and Georgios Tzimiropoulos \\ Kingston University and University of Nottingham
}

\begin{abstract}
We present a novel frequency-domain image registration technique, which employs histograms of oriented gradients providing subpixel estimates. Our method involves image filtering using dense Histogram of Oriented Gradients (HOG), which provides an advanced representation of the images coping with real-world registration problems such as non-overlapping regions and small deformations. The proposed representation retains the orientation information and the corresponding weights in a multi-dimensional representation. Furthermore, due to the overlapping local contrast normalization characteristic of $\mathrm{HOG}$, the proposed Histogram of Oriented Gradients - Phase Correlation (HOG-PC) method improves significantly the estimated motion parameters in small size blocks. Experiments using sequences with and without ground truth including both global and local/multiple motions demonstrate that the proposed method outperforms the state-of-the-art in frequency-domain motion estimation, in the shape of phase correlation, in terms of subpixel accuracy and motion compensation prediction for a range of test material, block sizes and motion scenarios. Keywords: Phase Correlation, registration in frequency domain, subpixel, Fourier, Histogram of Oriented Gradients.
\end{abstract}

\section{Introduction}

2 A critical component of various high-level computer vision and video pro3 cessing systems is motion estimation and registration. To perform image reg4 istration, we usually assume that the input images are related by a parametric 
geometrical transformation. Then, in order to obtain the unknown motion parameters, an optimisation approach is applied on a matching criterion. Pure translation is assumed in this work, which is fundamental in a number of applications such as standards conversion, noise reduction, image super-resolution, medical image registration, restoration, and compression. In such systems, motion compensated prediction is widely used for filtering and redundancy reduction purposes. International standards for video communications such as MPEGx and H.26x employ motion compensation prediction, which is based on regular block-based partitions of incoming frames.

Recently there has been a lot of interest in motion estimation techniques operating in the frequency domain. Perhaps the best-known method in this class is phase correlation $[1,2]$, which has become one of the motion estimation methods of choice for a wide range of professional studio and broadcasting applications [3]. Phase Correlation (PC) and other frequency domain approaches (that are based on the shift property of the Fourier Transform (FT)) offer speed through the use of FFT routines and enjoy a high degree of accuracy featuring several significant properties: immunity to uniform variations of illumination, insensitivity to changes in spectral energy and excellent peak localization accuracy. Furthermore, it provides sub-pixel accuracy that has a significant impact on motion compensated error performance and image registration for super-resolution and other applications, as theoretical and experimental analyses have suggested [4]. Sub-pixel accuracy mainly can be achieved through the use of interpolation, which is also applicable to frequency domain motion estimation methods.

One of the main issues of frequency domain registration methods is that in order to obtain reliable motion estimates large blocks of image data are required. Although this requirement is not an issue when there is a single motion, it causes problems when multiple motions are present and affects the accuracy and the overall motion compensated error (especially at the motion borders). On the other hand, reducing the block size increases the sensitivity to noise and reduces the amount of useful image information. Therefore to circumvent the problem, selecting useful and reliable features is essential. In computer vision and image 
processing, histogram of oriented gradients (HOG) [5] is a feature descriptor that is invariant to geometric and photometric transformations used mainly for object recognition. Histogram of oriented gradients describe local shapes within an image by the distribution of intensity gradients. The image is divided into cells, and for the pixels within each cell, a histogram of gradient directions is calculated. The local histograms can be normalized by calculating a measure of the intensity across a larger block over a set of neighbouring cells providing invariance to changes in illumination and shadowing.

The main point of this work is to propose a dense HOG-based PC method that is invariant to small deformations, and performs well when the assumption for translation invariance breaks. To the best of our knowledge this is one of the most important problems in block-based motion estimation, as the problem of noise has been addressed by many authors in the past. Additionally, the limitations of frequency based methods when small blocks are used is key part of the motivation of the combination, since HOG transform provides an extra advantage in very small block sizes. In more details, in this paper we introduce a novel high-performance version of the phase correlation algorithm based on histogram of oriented gradients (HOG-PC). The key advances introduced by this paper are the use of a dense histogram of oriented gradients to represent the images. Note that the proposed dense representation is quite different from the traditional representation of a block (or patch) based on HOG. The latter achieves invariance to small translational displacements and hence does not appear to be suitable for motion estimation. In contrast, we propose to use a very dense representation by calculating a descriptor per pixel. This allows us to interpret the obtain representation as a multi-channel block representation. Then, motion estimation is performed by correlating the multi-channel representations from two blocks. Our main contribution lies in showing that this representation not only can recover translational motion very accurately but is also better able to cope with real-world registration problems such as non-overlapping regions, small deformations but also white noise. Furthermore, due to the overlapping local contrast normalization characteristic of HOG, the 
proposed HOG-PC method improves significantly the estimated motion parameters in smaller size blocks. Finally, subpixel accuracy is obtained through the use of simple interpolation schemes [6,7]. Experiments with ground truth data, noisy MR images, and real video sequences have shown that our scheme performs significantly better than recently proposed subpixel extensions to the phase correlation method.

This paper is organised as follows. In Section 2, we review the state-of-theart in sub-pixel motion estimation using phase correlation. In Section 3, we discuss the principles of the proposed HOG-PC and the key features of this method are analysed. In Section 4 we present experimental results while in Section 5 we draw conclusions arising from this paper.

\section{Related work}

In this section, a brief review of current state-of-the-art Fourier-based methods for image registration is presented [8]. In many practical encoder implementations, sub-pixel motion estimation is achieved by straightforward extensions to the baseline integer-pixel block-matching algorithm mainly through the use of interpolation. Interpolation in the data domain is also applicable to frequency domain motion estimation methods such as phase correlation. Moreover such an approach cannot provide estimates of true floating-point accuracy, only approximations to the nearest negative power of two. To circumvent the above difficulties associated with interpolation, alternative approaches have been developed.

Recently, several subpixel extensions have been proposed $[9,10,11,12,13$, 14]. In [15], Hoge proposes to perform the unwrapping after applying a rank-1 approximation to the phase difference matrix. In more detail, Hoge presents a so-called Subspace Identification Extension method, which is based on the observation that a 'noise-free' phase correlation matrix (i.e. a matrix computed from shifted replicas of the same image) is a rank one, separable-variable matrix. For a "noisy phase correlation matrix (i.e. a matrix computed from consecu- 

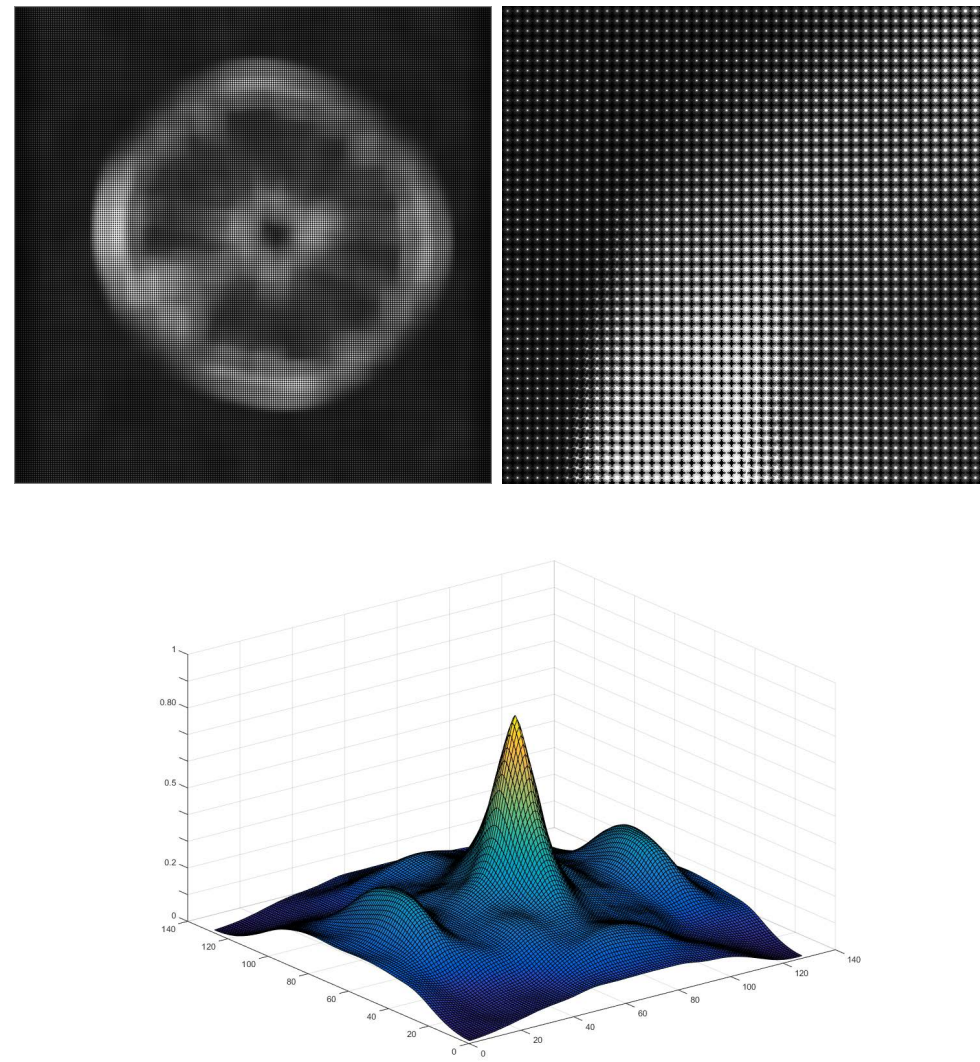

Figure 1: An example of the dense HOG features represented with orientation histograms (top) and a obtained correlation surface (bottom).

96 tive frames of a moving sequence), the sub-pixel motion estimation problem can be recast as finding the rank one approximation to that matrix. This can be achieved by using Singular Value Decomposition (SVD) followed by the identification of the left and right singular vectors. These vectors allow the construction of a set of normal equations, which can be solved to yield the required estimate. The work in [16] is a noise-robust extension to [15], where noise is assumed to be AWGN. The authors in [17] derive the exact parametric model of the phase difference matrix and solve an optimization problem for fitting the analytic model to the noisy data.

To estimate the subpixel shifts, Stone et al. [18] fit the phase values to a 2-D 

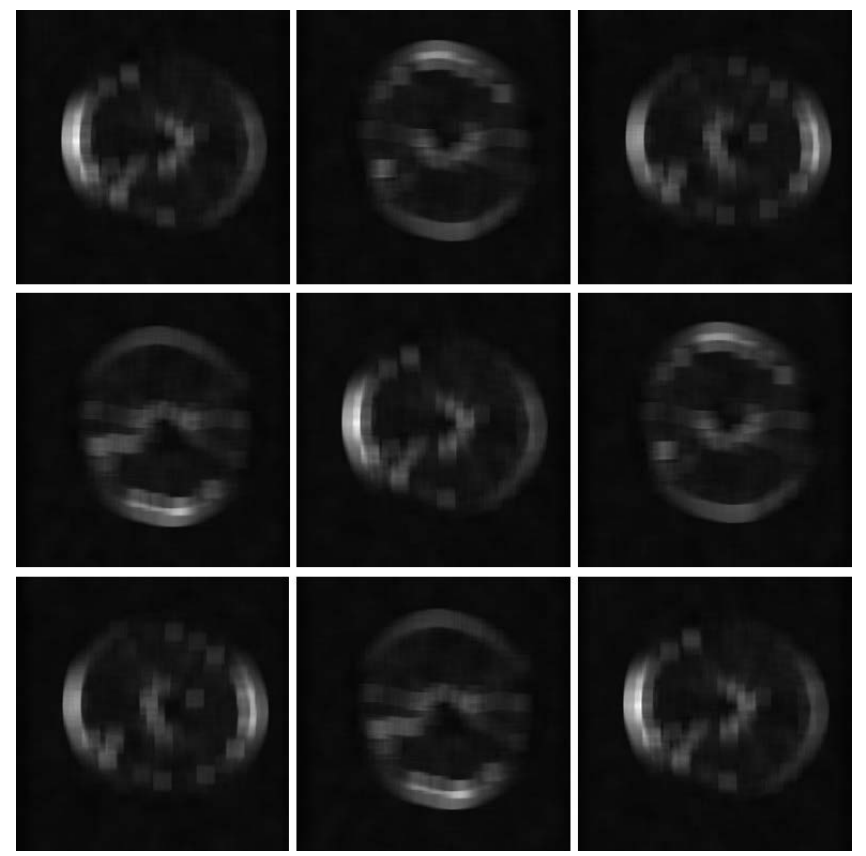

Figure 2: The first $\theta=9$ channels of the dense HOG that were used in the proposed HOG-PC.

linear function using linear regression, after masking out frequency components corrupted by aliasing. The method inevitably requires 2-D phase unwrapping which is a difficult ill-posed problem, while the parameters controlling masking are arbitrarily chosen and require fine tuning. Thus, after obtaining an integerprecision alignment of the input images their method takes steps towards alias cancellation by eliminating certain spectral components of each of the two input images. Elimination is based on two criteria: (i) radial distance of a spectral component from the component located at the origin and (ii) magnitude of a spectral component in relation to a threshold. The latter is dynamically determined as follows. Spectral components are sorted by magnitude and are progressively eliminated starting with the lowest. The authors claim that there exists a range in which the accuracy of the computed motion estimate becomes stable and independent of the degree of progressive elimination. This stability range is indirectly used to determine the required threshold. A plane fitting operation on the frequencies that have survived the above two criteria yields 


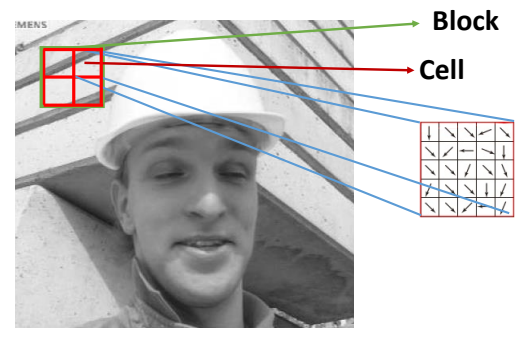

Figure 3: An example of a block and its cells used in HOG transform estimation.

the required motion estimates. An extension to the method for the additional estimation of planar rotation has been proposed in [19].

Foroosh et al. [20] showed that the phase correlation function is the Dirichlet kernel and provided analytic results for the estimation of the subpixel shifts using the sinc approximation. According to [20], images mutually shifted by a sub-pixel amount can be assumed as having been obtained by an integer pixel displacement on a higher resolution grid followed by subsampling. This assumption allows the analytic computation of the normalised cross-power spectrum as a polyphase decomposition of a filtered unit impulse. The authors demonstrate that the signal power of the resulting phase correlation surface is not concentrated in a single peak but is distributed to several coherent peaks adjacent to each other. The authors further show that this amounts to a Dirichlet kernel, which can be closely approximated by a sinc function. This approximation allows for the development of a closed-form solution for the sub-pixel shift estimate.

Finally, a fast method for subpixel estimation based on FFTs has been proposed in [21]. Notice that the above methods either assume aliasing-free images $[20,22,21,17]$, or cope with aliasing by frequency masking $[18,16,15,19]$, which requires fine tuning. 

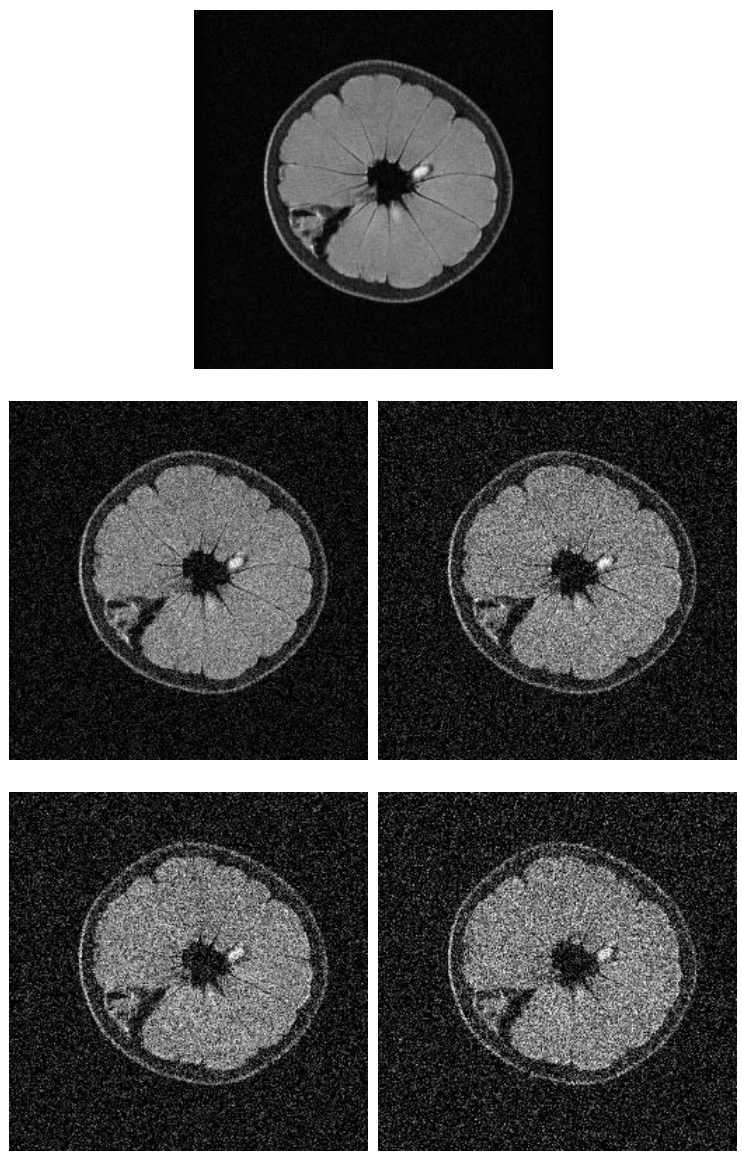

Figure 4: An example of the MRI data without and with noise of different levels $(0.01,0.02,0.03,0.04)$.

\section{HOG-PC for Subpixel Registration}

141

Let $I_{i}(\mathbf{x}), \mathbf{x}=[x, y]^{T} \in \mathcal{R}^{2}, i=1,2$ be two image functions, related by an unknown translation $\mathbf{t}=\left[t_{x}, t_{y}\right]^{T} \in \mathcal{R}^{2}$

$$
I_{2}(\mathbf{x})=I_{1}(\mathbf{x}-\mathbf{t})
$$

${ }_{143}$ To estimate the translational displacement, we use phase based correlation 144 schemes. Each image $I_{i}(\mathbf{x})$ can be considered as a continuous periodic im145 age function with period $T_{x}=T_{y}=1$, [23]. The Fourier series coefficients of $I$ 

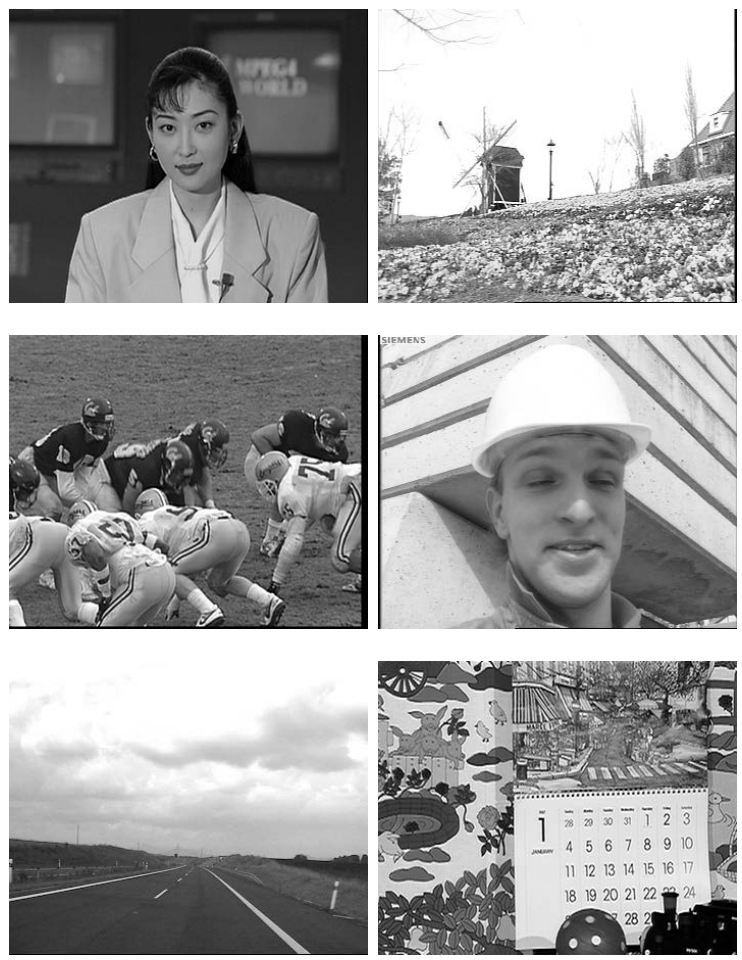

Figure 5: A frame of each video sequence that was used in our evaluation process.

146 are given by

$$
F_{I}(\mathbf{k})=\int_{\Omega} I(\mathbf{x}) e^{-j \omega_{0} \mathbf{k}^{T} \mathbf{x}} d \mathbf{x}
$$

147 where $\Omega=\{\mathbf{x}:-1 / 2 \leq \mathbf{x} \leq 1 / 2\}, \mathbf{k}=[k, l]^{T} \in Z^{2}$ and $\omega_{0}=2 \pi$. If we sample

${ }_{148} I$ at a rate $N$ with a $2-D$ Dirac comb function $D(\mathbf{x})=\sum_{\mathbf{s}} \delta(\mathbf{x}-\mathbf{s} / N)$, we obtain

149 a set of $N \times N$ discrete image values $I_{1}(\mathbf{m})=I(\mathbf{m} / N), \mathbf{m}=[m, n]^{T} \in \mathcal{Z}^{2}$ and

${ }_{150}-N / 2 \leq \mathbf{m}<N / 2,[23]$. Using $D$, we can write the DFT of $I_{1}$ as 
Table 1: Average PSNR (dB) values for all the video sequences and block size $8 \times 8$.

\begin{tabular}{c|cccccccc}
\hline \hline Method & GC & NGC & HOGPC & Hoge & Foroosh & Xiaohua & PC & Ren \\
\hline Akiyo & 42.4208 & 43.0771 & $\mathbf{4 3 . 2 2 0 1}$ & 39.8234 & 40.8600 & 40.9810 & 38.8748 & 41.7708 \\
Flower & 23.6991 & 25.2763 & $\mathbf{2 6 . 0 8 1 5}$ & 17.5719 & 19.8677 & 20.0458 & 15.9774 & 20.5315 \\
Football & 18.7215 & 18.7584 & $\mathbf{1 8 . 8 0 4 0}$ & 17.9245 & 18.2426 & 18.2003 & 17.7605 & 18.3034 \\
Foreman & 27.5536 & 28.3746 & $\mathbf{2 8 . 6 0 8 8}$ & 24.6746 & 26.1103 & 26.0809 & 24.2605 & 35.9339 \\
Highway & 31.5640 & 32.1402 & $\mathbf{3 2 . 3 3 6 2}$ & 30.4101 & 31.2490 & 30.9609 & 29.0454 & 32.8063 \\
MobCal & 21.6909 & 23.5442 & $\mathbf{2 3 . 9 3 4 9}$ & 16.6177 & 19.3245 & 19.4276 & 14.9527 & 21.8954 \\
\hline
\end{tabular}

$$
\begin{array}{r}
\hat{I}_{1}(\mathbf{k})=\sum_{\mathbf{m}} I_{1}(\mathbf{m}) e^{-j(2 \pi / N) \mathbf{k}^{T} \mathbf{m}} \\
=\int_{\Omega} D(\mathbf{x}) I(\mathbf{x}) e^{-j(2 \pi / N) \mathbf{k}^{T} \mathbf{x}} d \mathbf{x} \\
=F_{I}(\mathbf{k}) \star \sum_{\mathbf{s}} e^{-j(2 \pi / N) \mathbf{k}^{T} \mathbf{s} / N} \\
=N^{2} \sum_{\mathbf{s}} F_{I}(\mathbf{k}-\mathbf{s} N)
\end{array}
$$

where $-N / 2 \leq \mathbf{k}<N / 2$ and $\star$ denotes convolution.

Moving to the shifted version of the image [23], given by the equation (1) with $\mathbf{t}=\left[t_{x}, t_{y}\right]^{T},\{\mathbf{t}:-1<N \mathbf{t}<1\}$. Sampling with $D$ in a similar fashion we get $I_{2}$ and its DFT is given based on the Fourier shift property by

$$
\hat{I}_{2}(\mathbf{k})=N^{2} \sum_{\mathbf{s}} F_{I}(\mathbf{k}-\mathbf{s} N) e^{-j(2 \pi / N)(\mathbf{k}-\mathbf{s} N)^{T}(N \mathbf{t})}
$$

Assuming no aliasing and combining equations (3) and (4) we have

$$
\hat{I}_{2}(\mathbf{k})=\hat{I}_{1}(\mathbf{k}) e^{-j(2 \pi / N) \mathbf{k}^{T}(N \mathbf{t})}
$$

Note that the well-known shift property of the DFT refers to integer shifts and does not assume aliasing-free signals. Hereafter, we assume that our sampling device eliminates aliasing. Traditionally to estimate the translational displacement, we use phase correlation (PC), which is perhaps the most widely used correlation-based method in image registration. It looks for the maximum of the phase difference function which is defined as the inverse FT of the 
Table 2: Average PSNR (dB) values for all the video sequences and block size $16 \times 16$.

\begin{tabular}{c|cccccccc}
\hline \hline Method & GC & NGC & HOGPC & Hoge & Foroosh & Xiaohua & PC & Ren \\
\hline Akiyo & 43.1677 & $\mathbf{4 3 . 2 0 9 4}$ & 43.1455 & 43.1980 & 41.4381 & 41.8490 & 41.2989 & 41.0237 \\
Flower & 28.3028 & 28.5663 & $\mathbf{2 8 . 7 0 7 6}$ & 23.8995 & 25.9038 & 25.3230 & 15.7029 & 24.4162 \\
Football & 19.6813 & 19.7728 & $\mathbf{2 0 . 0 3 3 8}$ & 18.1636 & 18.6105 & 18.4471 & 17.6603 & 18.5527 \\
Foreman & 29.5387 & 29.7872 & $\mathbf{3 0 . 2 1 9 2}$ & 25.9039 & 27.3208 & 28.0282 & 24.2521 & 36.3688 \\
Highway & 32.5355 & 32.8818 & $\mathbf{3 3 . 6 1 6 6}$ & 31.3321 & 31.7945 & 30.7668 & 28.5041 & 32.8148 \\
MobCal & 24.5285 & 24.8592 & $\mathbf{2 4 . 9 1 0 1}$ & 21.7679 & 23.0760 & 23.6421 & 14.6892 & 22.7079 \\
\hline
\end{tabular}

Table 3: Average PSNR (dB) values for all the video sequences and block size $32 \times 32$.

\begin{tabular}{c|cccccccc}
\hline \hline Method & GC & NGC & HOGPC & Hoge & Foroosh & Xiaohua & PC & Ren \\
\hline Akiyo & 42.1412 & $\mathbf{4 2 . 1 5 5 4}$ & 41.9947 & 41.0056 & 39.8609 & 41.4001 & 38.2170 & 40.5871 \\
Flower & 28.3463 & $\mathbf{2 8 . 3 8 9 4}$ & 28.3483 & 27.0887 & 27.8201 & 27.1752 & 15.6074 & 25.6091 \\
Football & 20.4393 & 20.5912 & $\mathbf{2 0 . 7 8 3 2}$ & 18.7080 & 19.5177 & 18.9756 & 17.5200 & 18.6555 \\
Foreman & 30.7678 & 31.1017 & $\mathbf{3 1 . 4 6 7 3}$ & 27.6488 & 29.5744 & 29.4516 & 24.2495 & 36.2586 \\
Highway & 32.9365 & 33.1902 & $\mathbf{3 3 . 8 0 9 9}$ & 32.1031 & 32.3265 & 29.5379 & 28.5122 & 33.8376 \\
MobCal & 24.1923 & $\mathbf{2 4 . 2 2 4 1}$ & 24.1245 & 23.7131 & 23.5444 & 23.5672 & 14.5033 & 23.4697 \\
\hline
\end{tabular}

normalized cross-power spectrum [1]

$$
\mathrm{PC}(\mathbf{u}) \triangleq F^{-1}\left\{\frac{\hat{I}_{2}(\mathbf{k}) \hat{I}_{1}^{*}(\mathbf{k})}{\left|\hat{I}_{2}(\mathbf{k})\right|\left|\hat{I}_{1}^{*}(\mathbf{k})\right|}\right\}=F^{-1}\left\{e^{j \mathbf{k}^{T} \mathbf{t}}\right\}=\delta(\mathbf{u}-\mathbf{t})
$$

163

where $*$ denotes complex conjugate and $F^{-1}$ the inverse Fourier transform. Regarding the differences with the work in [23] the two main ones are highlighted. The first is in the data representation used for motion estimation. In the proposed approached a dense HOG is introduced as a representation that is invariant to small deformations and hence robust when the assumed translational motion model breaks (e.g. video block matching). Also the approach in [23] uses image gradients which do not possess this property. The second is in contrary to [23], we found that our method does not benefit from the rank-1 approximation to the correlation function. 
Table 4: Average PSNR (dB) values for all the video sequences (50 first frames) and block size $8 \times 8$ with 0.75 variance motion blur.

\begin{tabular}{c|cccccccc}
\hline \hline Method & GC & NGC & HOGPC & Hoge & Foroosh & Xiaohua & PC & Ren \\
\hline Akiyo & 46.9358 & 47.9122 & $\mathbf{4 8 . 1 8 9 4}$ & 43.8985 & 44.0877 & 43.5653 & 43.4136 & 46.3062 \\
Flower & 28.4947 & 30.4891 & $\mathbf{3 1 . 5 6 2 9}$ & 22.6101 & 24.8113 & 22.4272 & 21.7711 & 26.0737 \\
Football & 18.2997 & 18.2785 & $\mathbf{1 8 . 3 1 2 6}$ & 17.3936 & 17.6461 & 17.3321 & 17.3271 & 17.8680 \\
Foreman & 31.4301 & 32.5690 & $\mathbf{3 3 . 3 0 0 6}$ & 28.3002 & 29.1089 & 28.1744 & 27.9264 & 30.5642 \\
Highway & 36.2005 & 36.8582 & $\mathbf{3 7 . 1 5 5 1}$ & 34.6884 & 35.0371 & 34.3573 & 33.5344 & 36.0504 \\
MobCal & 25.3502 & 27.3564 & $\mathbf{2 7 . 6 5 3 1}$ & 20.4433 & 22.0091 & 20.0361 & 19.5922 & 23.6230 \\
\hline
\end{tabular}

Table 5: Average PSNR (dB) values for all the video sequences (50 first frames) and block size $16 \times 16$ with 0.75 variance motion blur.

\begin{tabular}{c|cccccccc}
\hline \hline Method & GC & NGC & HOGPC & Hoge & Foroosh & Xiaohua & PC & Ren \\
\hline Akiyo & 47.7864 & 47.7916 & $\mathbf{4 7 . 8 8 6 9}$ & 44.5982 & 43.9858 & 44.7131 & 43.2949 & 46.1797 \\
Flower & 36.2843 & 36.8024 & $\mathbf{3 7 . 0 9 6 0}$ & 24.6397 & 28.5770 & 32.9379 & 21.2003 & 30.4665 \\
Football & 19.2769 & 19.3445 & $\mathbf{1 9 . 6 4 6 7}$ & 17.4480 & 17.6951 & 17.9852 & 17.2224 & 17.8443 \\
Foreman & 33.8094 & 33.8268 & $\mathbf{3 4 . 7 3 8 5}$ & 28.4528 & 28.8829 & 31.1467 & 27.7902 & 30.1301 \\
Highway & 37.0921 & 37.7399 & $\mathbf{3 8 . 3 7 9 4}$ & 35.4120 & 35.6794 & 34.8676 & 33.3544 & 36.5686 \\
MobCal & 29.0098 & 29.5434 & $\mathbf{2 9 . 7 6 3 0}$ & 22.9852 & 22.6976 & 26.2229 & 19.3175 & 24.6576 \\
\hline
\end{tabular}

\subsection{Proposed methodology for $H O G-P C$}

In this section, we introduce the proposed phase correlation algorithm based on histogram of oriented gradients (HOG-PC). Note that the proposed dense representation is quite different from the traditional representation of a block (or patch) based on HOG. The latter achieves invariance to small translational displacements and hence does not appear to be suitable for motion estimation. In contrast, we propose to use a very dense representation by calculating a descriptor per pixel. This allows us to interpret the obtain representation as a multi-channel block representation. Then, motion estimation is performed by correlating the multi-channel representations from two blocks. Our main contribution lies in showing that this representation not only can recover translational motion very accurately but is also better able to cope with real-world registration problems such as non-overlapping regions small deformations but also white 
Table 6: Average PSNR (dB) values for all the video sequences (50 first frames) and block size $32 \times 32$ with 0.75 variance motion blur.

\begin{tabular}{c|cccccccc}
\hline \hline Method & GC & NGC & HOGPC & Hoge & Foroosh & Xiaohua & PC & Ren \\
\hline Akiyo & $\mathbf{4 7 . 2 5 9 8}$ & 47.0844 & 46.9395 & 42.8979 & 43.2420 & 45.2531 & 42.8459 & 45.1704 \\
Flower & $\mathbf{3 7 . 7 4 7 1}$ & 37.7145 & 37.5289 & 34.4633 & 31.8037 & 35.6403 & 21.0538 & 33.7585 \\
Football & 20.0454 & 20.2501 & $\mathbf{2 0 . 4 6 7 6}$ & 17.9476 & 17.4846 & 18.2341 & 17.0028 & 17.5864 \\
Foreman & 34.4031 & 34.5012 & $\mathbf{3 5 . 1 4 4 8}$ & 29.4785 & 28.9129 & 32.9204 & 27.6481 & 29.9641 \\
Highway & 38.3238 & 38.8231 & $\mathbf{3 9 . 3 6 3 5}$ & 36.4025 & 36.3173 & 34.2663 & 33.3791 & 36.9849 \\
MobCal & 29.3901 & $\mathbf{2 9 . 4 7 4 1}$ & 29.3422 & 27.5164 & 23.2643 & 27.5576 & 18.9994 & 25.1790 \\
\hline
\end{tabular}

Table 7: Average PSNR (dB) values for all the video sequences (50 first frames) and block size $8 \times 8$ with 1.75 variance motion blur.

\begin{tabular}{c|cccccccc}
\hline \hline Method & GC & NGC & HOGPC & Hoge & Foroosh & Xiaohua & PC & Ren \\
\hline Akiyo & 50.2757 & 51.0925 & $\mathbf{5 1 . 3 3 9 0}$ & 47.4929 & 47.0069 & 47.3463 & 47.2890 & 49.0431 \\
Flower & 31.4674 & 32.8165 & $\mathbf{3 3 . 3 9 3 4}$ & 27.2786 & 27.6245 & 27.2046 & 26.9964 & 29.2145 \\
Football & $\mathbf{1 9 . 4 7 2 5}$ & 19.3277 & 19.3736 & 18.5606 & 18.6270 & 18.5354 & 18.5142 & 18.8796 \\
Foreman & 32.3824 & 33.2128 & $\mathbf{3 3 . 7 4 9 8}$ & 29.9563 & 29.9954 & 29.8935 & 29.6478 & 31.4852 \\
Highway & 38.8627 & 39.1613 & $\mathbf{3 9 . 4 5 7 1}$ & 37.5952 & 37.3573 & 37.5161 & 36.8784 & 38.2206 \\
MobCal & 28.1125 & 29.4835 & $\mathbf{2 9 . 6 9 7 6}$ & 24.4869 & 24.6221 & 24.3584 & 24.1381 & 26.2775 \\
\hline
\end{tabular}

noise. Furthermore, due to the overlapping local contrast normalization characteristic of HOG, the proposed HOG-PC method improves significantly the estimated motion parameters in smaller size blocks. Finally, subpixel accuracy is obtained through the use of simple interpolation schemes $[6,7]$.

We first describe the traditional HOG descriptor. HOG uses the normalized combination of gradient vectors from a given number of pixels to build up a histogram of binned angles that relate to the feature. The process begins by breaking the image up into set features spaces $f$ comprised of a number of cells $c$, which in turn is made up of pixels. In more details, the feature spaces are overlapping blocks in a dense manner and each one of them has $2 \times 2$ cells of size $8 \times 8$ pixels (see figure 3 ). For each pixel within a cell the filter mask $[-1,0,1]$ is applied to its neighbouring pixels giving us the gradient vector $\vec{g}$.

The magnitude $\|\vec{g}\|$ of the gradient vector is obtained and its orientation 
Table 8: Average PSNR (dB) values for all the video sequences (50 first frames) and block size $16 \times 16$ with 1.75 variance motion blur.

\begin{tabular}{c|cccccccc}
\hline \hline Method & GC & NGC & HOGPC & Hoge & Foroosh & Xiaohua & PC & Ren \\
\hline Akiyo & 51.4247 & 51.3765 & $\mathbf{5 1 . 6 4 8 5}$ & 47.3513 & 46.8294 & 50.0535 & 47.1346 & 48.8104 \\
Flower & 38.2331 & 39.0902 & $\mathbf{3 9 . 7 5 3 6}$ & 27.5034 & 27.9538 & 35.9841 & 27.0223 & 29.7296 \\
Football & 20.2406 & 20.3819 & $\mathbf{2 0 . 6 1 4 0}$ & 18.4652 & 18.5598 & 19.0224 & 18.4073 & 18.7527 \\
Foreman & 34.5928 & 34.2964 & $\mathbf{3 5 . 3 1 7 6}$ & 29.8665 & 29.7574 & 33.3645 & 29.6204 & 30.9343 \\
Highway & 40.1471 & 40.3989 & $\mathbf{4 1 . 0 3 0 1}$ & 37.7915 & 37.6942 & 38.7673 & 36.9909 & 38.5279 \\
MobCal & 31.6078 & 32.2799 & $\mathbf{3 2 . 4 2 3 6}$ & 24.8317 & 24.6213 & 29.8854 & 24.1093 & 26.2672 \\
\hline
\end{tabular}

Table 9: Average PSNR (dB) values for all the video sequences (50 first frames) and block size $32 \times 32$ with 1.75 variance motion blur.

\begin{tabular}{c|cccccccc}
\hline \hline Method & GC & NGC & HOGPC & Hoge & Foroosh & Xiaohua & PC & Ren \\
\hline Akiyo & $\mathbf{5 1 . 2 5 3 9}$ & 50.7153 & 50.6264 & 40.8783 & 46.4685 & 49.6346 & 46.6632 & 47.7458 \\
Flower & $\mathbf{4 3 . 1 4 8 3}$ & 42.6464 & 42.5386 & 30.6144 & 28.6057 & 41.1037 & 27.2853 & 30.8018 \\
Football & 21.1975 & 21.3593 & $\mathbf{2 1 . 7 7 3 1}$ & 18.5759 & 18.3757 & 18.6508 & 18.2522 & 18.5016 \\
Foreman & 35.4305 & 35.2909 & $\mathbf{3 6 . 2 7 9 6}$ & 29.6047 & 29.6118 & 33.8368 & 29.4914 & 30.6552 \\
Highway & 41.0594 & 41.7453 & $\mathbf{4 2 . 0 3 4 3}$ & 37.8387 & 38.3018 & 39.9322 & 37.2725 & 39.0443 \\
MobCal & $\mathbf{3 3 . 2 9 1 5}$ & 33.2234 & 33.2526 & 26.9309 & 24.4869 & 30.5041 & 24.1541 & 26.2379 \\
\hline
\end{tabular}

expressed using angle $\theta$.

$$
\theta=\tan ^{-1}\left(g_{y}, g_{x}\right)
$$

Additionally a weight $w$ is defined for each pixel, which is used to scale its contribution to its cell's histogram. This is given by the mean value of the pixels within a given 2D kernel indicating the density over this area. By applying this weight, the proposed approach provides accurate estimates also in the presence of noise.

Once these values are established the pixels within each cell are binned into a histogram $H$ according to their $\theta$ angle. The value added to a bin is given as the weighted magnitude of the vector $w\|\vec{g}\|$. Finally all cell histograms within a multi-dimensional feature $H_{j}$ are normalised using the $L_{2}$ norm. 

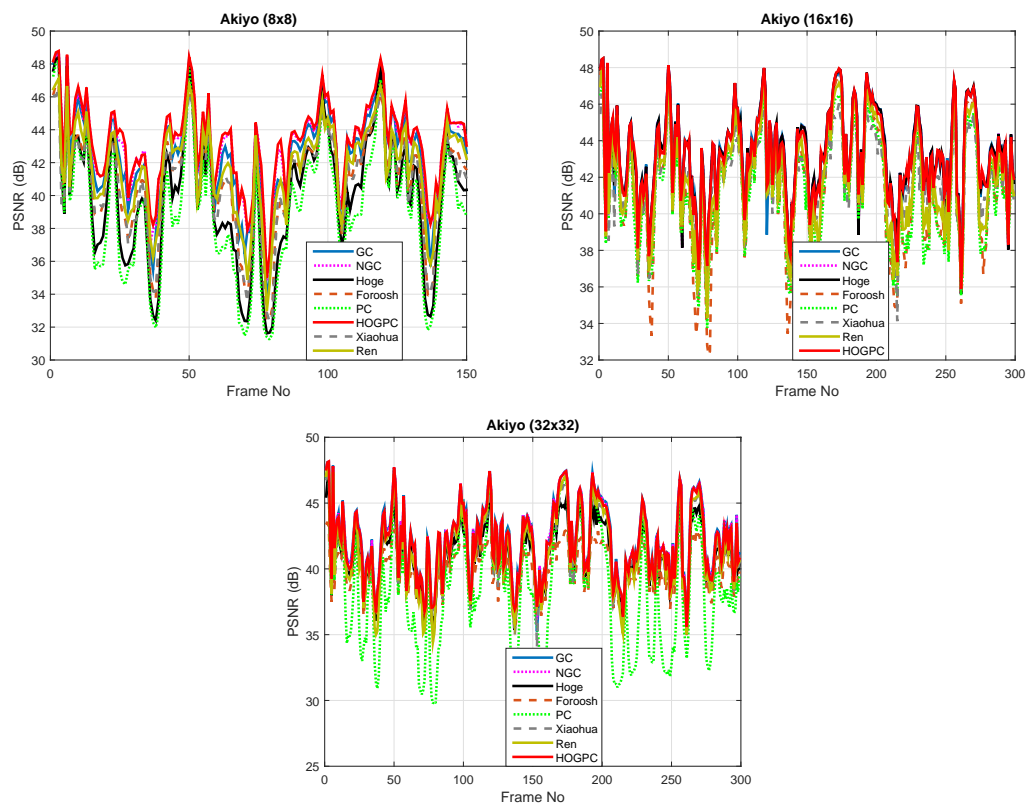

Figure 6: The PSNR values for the Akiyo sequence versus the frame number for all the block sizes.

$$
H_{j} \rightarrow \frac{H_{j}}{\sqrt{\left\|g_{\max }\right\|_{2}^{2}+e^{2}}}
$$

The obtained features are then vectorised as a $\theta$-dimensional descriptor

$$
\vec{d}=\left\{H_{1}, \ldots, H_{\theta}\right\}
$$

211 212

In this case the $\theta$-dimensional descriptor refers to the number of bins at the histogram with each one of these bins to correspond to an angle range.

Having defined HOG for a single cell, we now turn to the proposed dense HOG representation. For $I_{i}, i=1,2$, we extract $d$ from (9) at each pixel location $I_{i}(\mathbf{m})$ :

$$
H_{i}(\mathbf{m})=\left\{H_{i, 1}(\mathbf{m}), H_{i, 2}(\mathbf{m}), \ldots, H_{i, \theta}(\mathbf{m})\right\}
$$

The resulting histograms can be re-arranged as a multi-channel feature representation (see figures 1 and 2). 

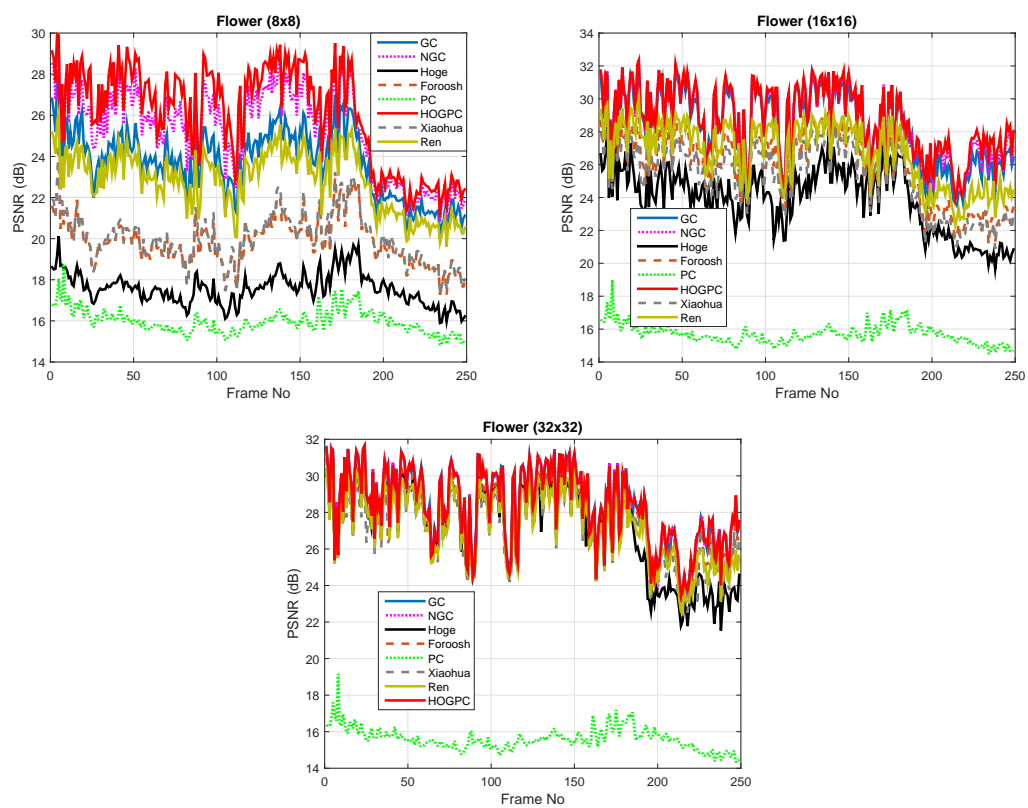

Figure 7: The PSNR values for the Flower sequence versus the frame number for all the block sizes.

$$
K_{1 D}\left(x ;\left\{x_{0}, \mathbf{p}\right\}\right)=p_{1}\left\{1-\left(p_{2}\left(x-x_{0}\right)\right)^{2}\right\} \frac{1}{\sqrt{2 \pi} p_{3}} e^{\frac{-\left(x-x_{0}\right)^{2}}{2 p_{3}^{2}}}
$$

which is a simple modification of the mexican hat wavelet [24]. To estimate $y_{0}$,

and find $\mathbf{t}=\arg \max _{\mathbf{m}} H O G P C(\mathbf{m})$. We can estimate sub-pixel accuracy registration $\mathbf{t}_{0}=\left(x_{0}, y_{0}\right)$ by fitting a $1 D$ kernel to the vicinity of the maximum on the correlation surface. A parametric kernel is used, which can adapt its shape to fit the correlation functions as well as to provide accurate estimates of the subpixel shifts. Based on the work in [23] a reasonable choice for our kernel is given by 

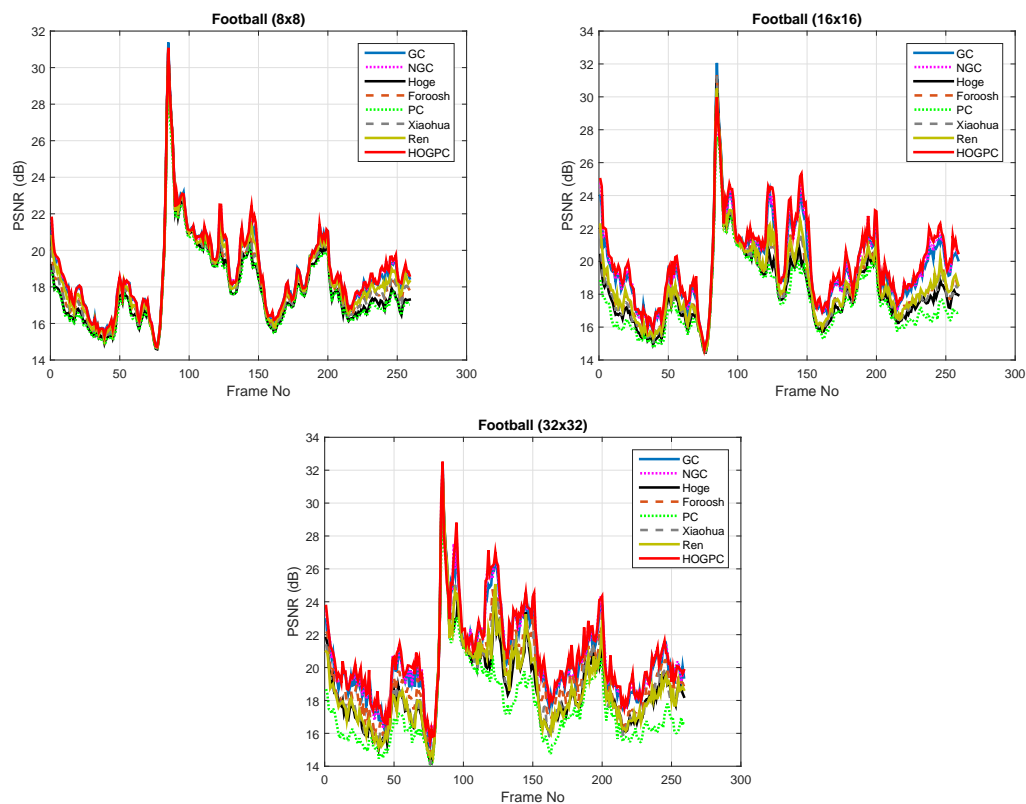

Figure 8: The PSNR values for the Football sequence versus the frame number for all the block sizes.

we set up a similar problem with the kernel defined as

$$
K_{1 D}\left(y ;\left\{y_{0}, \mathbf{q}\right\}\right)=q_{1}\left\{1-\left(q_{2}\left(y-y_{0}\right)\right)^{2}\right\} \frac{1}{\sqrt{2 \pi} q_{3}} e^{\frac{-\left(y-y_{0}\right)^{2}}{2 q_{3}^{2}}}
$$

Our algorithm estimates the kernel parameters $\left\{x_{0}, \mathbf{p}=\left[p_{1}, p_{2}, p_{3}\right]^{T}\right\}$ and $\left\{y_{0}, \mathbf{q}=\right.$ $\left.\left[q_{1}, q_{2}, q_{3}\right]^{T}\right\}$ in a least-squares sense. In more details, the kernel including the subpixel shift $\left(x_{0}, y_{0}\right)$ to be estimated is defined in the continuous domain, hence it allows (in both theory and practice) for the estimation of any subpixel shift. The choice of the kernel is related to the shape of the dense HOG correlation function. We found that the Mexican hat wavelet provides a good approximation to the underlying function enabling in practice the very accurate estimation of the subpixel shifts. 

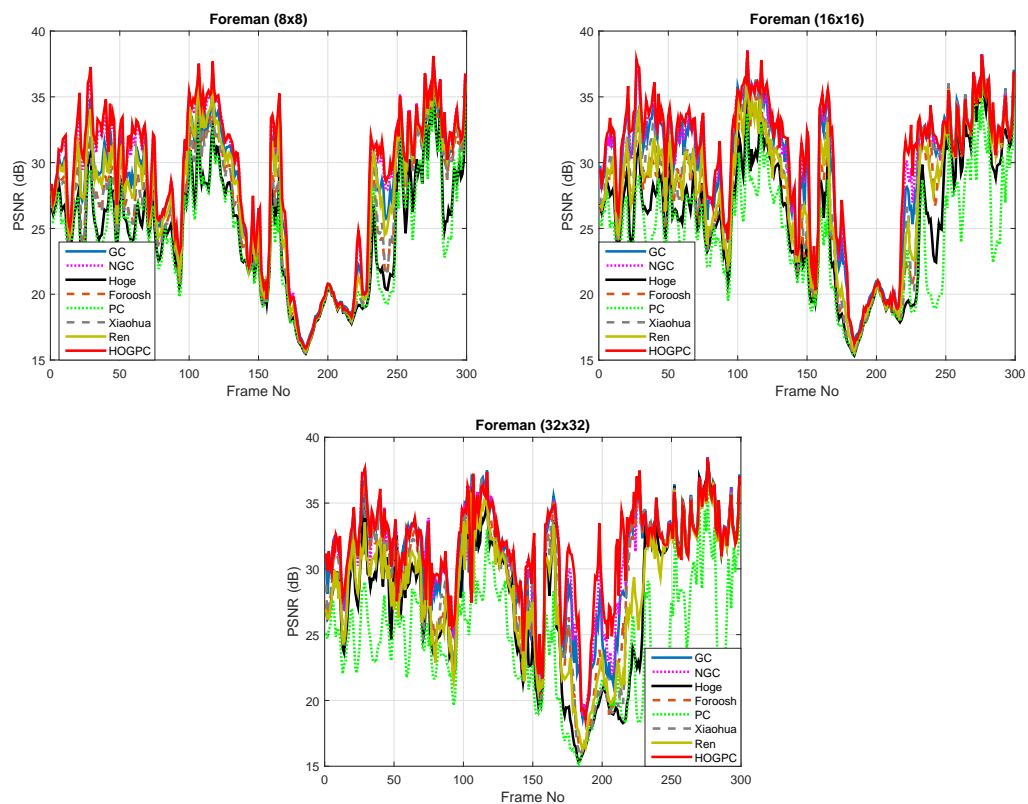

Figure 9: The PSNR values for the Foreman sequence versus the frame number for all the block sizes.

\section{Results}

To evaluate and illustrate the efficiency of the proposed scheme a comparative study was performed with state of the art frequency domain based techniques. Both data with ground truth and video sequences have been used for evaluating the performance. A set of MRI images are employed which have undergone sub-pixel displacement and it is available by the authors in [15] (see figure 4). The images show real MRI data from a grapefruit that was acquired using a production quality Fast Spin Echo (FSE) sequence on a GE (Faireld, CT, USA) Signa Lx 1.5 Tesla MRI scanner. The $256 \times 256$ pixel images cover a $16 \mathrm{~cm}^{2}$ FOV corresponding to a $0.0625 \mathrm{~mm}$ square per pixel. Five images were acquired with the fruit at dierent positions in the FOV, by manually moving the scanner table. Regarding the real videos the well-known sequences of 'Akiyo', 'Flower', 'Football', 'Foreman', 'Highway' and 'MobCal' were used including $150-300$ frames each (see figure 5). 

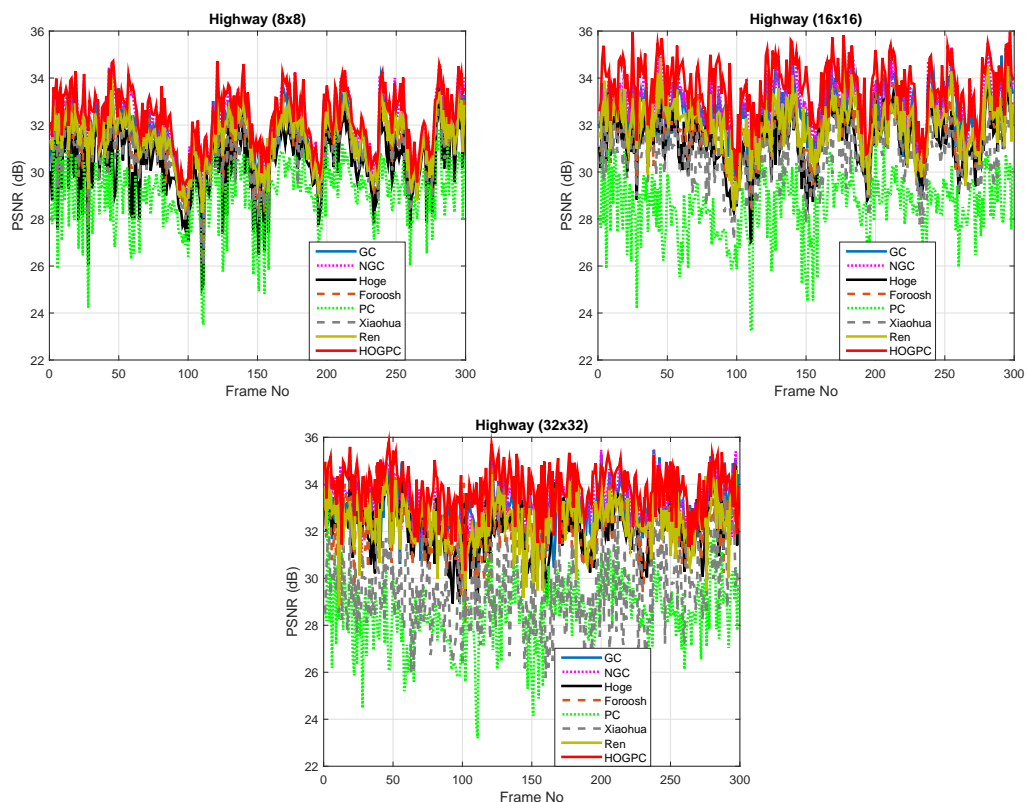

Figure 10: The PSNR values for the Highway sequence versus the frame number for all the block sizes.

\subsection{Video sequences without ground truth}

Regarding the real video sequences without ground truth, in order to evaluate the accuracy of the proposed method the visual quality (fidelity) of the motion compensated sequence is considered. It is defined as the closeness between the motion compensated frames and the original ones, and the peak signal to noise ratio (PSNR) is used in this work defined by

$$
P S N R=10 \log \left(\frac{255^{2}}{M S E_{I}}\right)
$$

where $M S E_{I}$ is the mean square error of the original and motion compensated frames.

The performance of the proposed $H O G P C$ scheme is compared with more than five popular $P C$ based methods $[15,20,22,6,17,7,23,9]$. Foroosh's method [20] estimates the subpixel shifts by fitting a sinc function to the available correlation samples. Hoge's and Xiaohua's [15, 14] methods are based on 

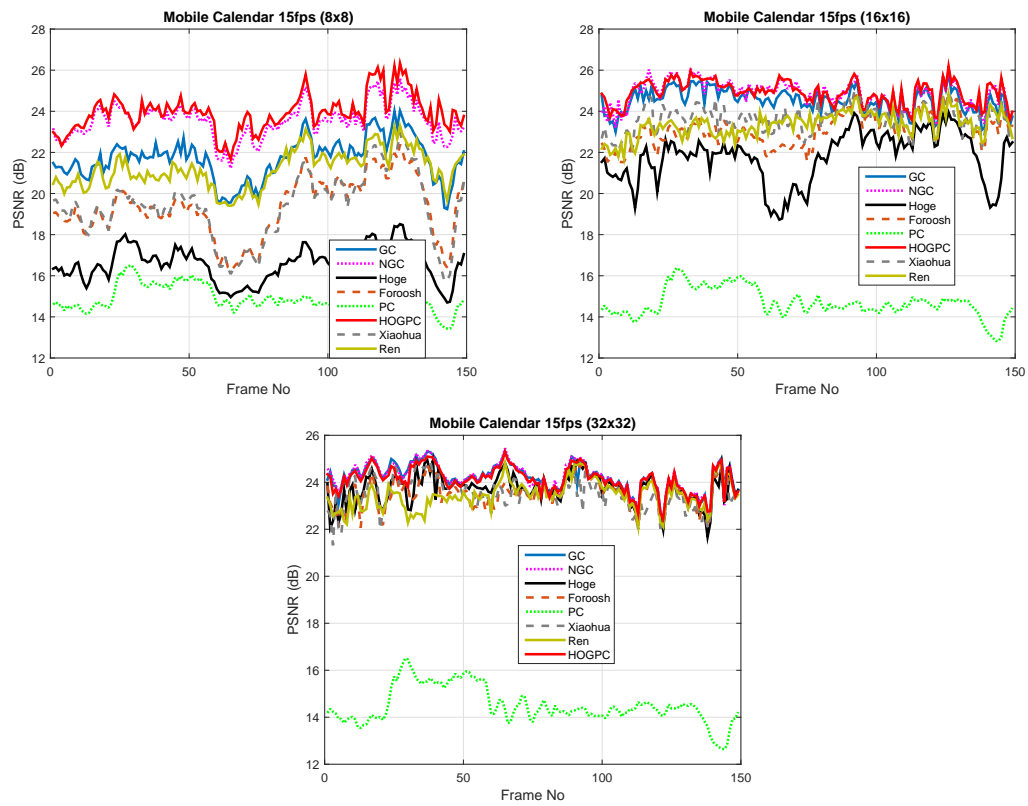

Figure 11: The PSNR values for the MobCal sequence versus the frame number for all the block sizes.

frequency masking, phase unwrapping and linear regression, while Ren's [22] approach applies a linear weighting of the height of the main peak on the one hand and the difference between its two neighboring side-peaks on the other.

In the second part of our evaluation process, experiments were performed using read video sequences and applying block based motion estimation. The selected block sizes were $32 \times 32,16 \times 16$ and $8 \times 8$ pixels and the motion compensated prediction error was estimated for each block size over all the sequences. The average PSNR values are shown in Tables 1,2 and 3 and it can be observed that the proposed approach results the highest values indicating better visual quality. In figures $6,7,8,9,10$, and 11 the PSNR values over time for the video sequences are shown with the proposed scheme to be the most accurate and consistent in comparison with the other state-of-the-art methods. Furthermore, experiments with motion blur present were performed indicating the accuracy of the proposed method especially in the case of small block sizes 


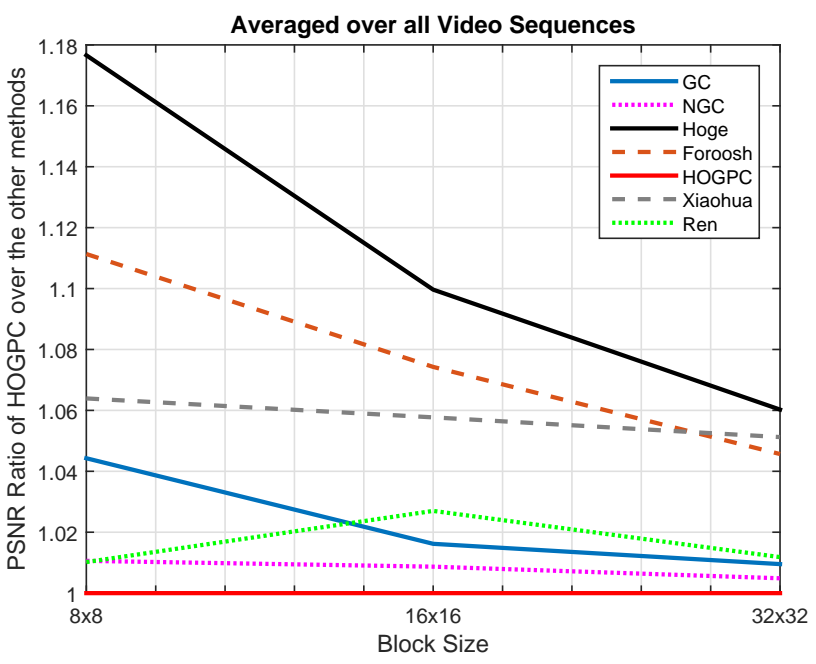

Figure 12: The PSNR ratio of the proposed HOGPC scheme over all the other methods for the different block sizes.

(e.g. $8 \times 8$ ). The average PSNR values are shown in Tables 4,5 and 6 for motion blur variance equal to 0.75 and in Tables 7,8 and 9 for motion blur variance equal to 1.75 .

Finally, in figure 12 we can see the gain of the HOGPC method as a ratio over the other approaches moving from larger to smaller block sizes. As it was expected the ratio increases due to the characteristics of our scheme and HOG. So, since HOG is utilising neighboring information (i.e. surrounding cells) even for small blocks $H O G P C$ scheme contains more information allowing more accurate estimates especially if larger motions are present. Furthermore, observing the results in Tables 1,2 and 3 focusing on the proposed method and especially for the Akiyo sequence that is characterised of small motion vectors in average, it shows that HOGPC provides the best results for the case of $8 \times 8$ pixels. Also, it outperforms other methods used over larger blocks such as $16 \times 16$ pixels, indicating the accuracy of the proposed HOGPC method that exploits the overlapping local contrast normalization characteristic of dense HOG. 
Table 10: Average MSE with the corresponding PSNR values, and the estimated motion vectors for the 10 image pairs of the MRI data (Part 1).

\begin{tabular}{c|cccc}
\hline \hline Image pairs & {$[1,2]$} & {$[1,3]$} & {$[1,4]$} & {$[1,5]$} \\
\hline GT & $(-2.40,4.00)$ & $(-4.80,8.00)$ & $(-7.20,4.32)$ & $(-7.20,12.00)$ \\
Hoge & $(-2.03,4.01)$ & $(-4.13,8.01)$ & $\mathbf{( - 6 . 8 1 , 4 . 1 7 )}$ & $\mathbf{( - 6 . 8 2 , 1 2 . 0 2 )}$ \\
Foroosh & $(\mathbf{- 2 . 2 2 , 4 . 2 3 )}$ & $(-4.36,8.24)$ & $(-6.59,4.41)$ & $(-6.59,12.26)$ \\
Balci & $(-2.11,4.10)$ & $(-3.90,8.05)$ & $(-6.22,4.34)$ & $(-6.39,12.15)$ \\
Gaussian & $(-2.07,4.02)$ & $(-4.33,8.01)$ & $(-6.57,4.37)$ & $(-6.57,12.06)$ \\
Quadratic & $(-2.03,4.01)$ & $(-4.18,8.00)$ & $(-6.73,4.25)$ & $(-6.74,12.03)$ \\
Sinc & $(-2.00,4.00)$ & $(-4.12,8.00)$ & $(-6.72,4.12)$ & $(-6.73,12.00)$ \\
ESinc & $(-2.00,4.00)$ & $(-4.25,8.00)$ & $(-6.54,4.31)$ & $(-6.54,12.04)$ \\
Ren & $(-2.09,4.02)$ & $(-4.34,8.01)$ & $(-6.58,4.38)$ & $(-6.59,12.08)$ \\
GC & $(-2.04,4.02)$ & $(-4.24,8.00)$ & $(-6.67,4.30)$ & $(-6.68,12.03)$ \\
NGC & $(-2.04,4.02)$ & $(-4.24,8.00)$ & $(-6.67,4.30)$ & $(-6.68,12.02)$ \\
Xiaohua & $(-2.04,3.95)$ & $(-4.23,7.97)$ & $(-6.66,4.36)$ & $(-6.68,12.06)$ \\
HOGPC & $(-2.06,4.04)$ & $(-4.25,8.03)$ & $(-6.67,4.33)$ & $(-6.67,12.04)$ \\
\hline
\end{tabular}

4.2. Real data with ground truth

In the case that ground truth is available, the mean square error (MSE) between the estimated subpixel motion vectors and the ground truth is used as a performance measure. Considering two vectors $\mathbf{u}$ and $\mathbf{v}$ representing the original (ground truth) and the estimated one, respectively, then

$$
M S E_{M V}=\frac{1}{n} \sum_{i=x, y}\left(u_{i}-v_{i}\right)^{2}
$$

where $n$ is the number of blocks in the frame. Consequently, a good quality estimate is expected to minimize MSE, which provides the accuracy of the estimates.

In more details, a set of five $256 \times 256$ pixel real MR images [15] was used and a sample of them is shown in figure 4 . The 5 images yield a total of 10 
Table 11: Average MSE with the corresponding PSNR values, and the estimated motion vectors for the 10 image pairs of the MRI data (Part 2).

\begin{tabular}{c|cccc}
\hline \hline Image pairs & {$[2,3]$} & {$[2,4]$} & {$[2,5]$} & {$[3,4]$} \\
\hline GT & $(-2.40,4.00)$ & $(-4.80,0.32)$ & $(-4.80,8.00)$ & $(-2.40,-3.68)$ \\
Hoge & $(-2.10,3.99)$ & $(-4.28,0.15)$ & $(-4.78,8.00)$ & $(-2.17,-3.84)$ \\
Foroosh & $(-2.32,3.75)$ & $(-4.55,0.39)$ & $(-4.55,8.24)$ & $(-2.40,-3.61)$ \\
Balci & $(-2.18,3.86)$ & $(-4.16,0.30)$ & $(-4.13,7.92)$ & $(-2.34,-3.62)$ \\
Gaussian & $(-2.26,3.97)$ & $(-4.55,0.35)$ & $(-4.56,8.01)$ & $(-2.43,-3.66)$ \\
Quadratic & $(-2.13,3.98)$ & $\mathbf{( - 4 . 6 5 , 0 . 2 2 )}$ & $(-4.65,8.00)$ & $(-2.25,-3.78)$ \\
Sinc & $(-2.09,4.00)$ & $(-4.72,0.11)$ & $(-4.71,8.00)$ & $(-2.27,-3.89)$ \\
ESinc & $(-2.19,4.00)$ & $(-4.59,0.28)$ & $(-4.60,8.00)$ & $(-2.46,-3.72)$ \\
Ren & $(-2.27,3.96)$ & $(-4.54,0.36)$ & $(-4.54,8.01)$ & $(-2.40,-3.65)$ \\
GC & $(-2.17,3.98)$ & $(-4.59,0.27)$ & $(-4.60,8.00)$ & $(-2.31,-3.71)$ \\
NGC & $(-2.17,3.98)$ & $(-4.59,0.27)$ & $(-4.60,8.00)$ & $(-2.31,-3.71)$ \\
Xiaohua & $(-2.18,3.96)$ & $(-4.59,0.34)$ & $(-4.58,8.04)$ & $(-2.39,-3.64)$ \\
HOGPC & $(-2.19,3.99)$ & $(-4.60,0.28)$ & $(-4.60,8.00)$ & $(-2.35,-3.68)$ \\
\hline
\end{tabular}

possible pairwise registrations and the ground truth of the subpixel translations is provided.

The estimated shifts and the corresponding measurements of their average MSE are shown in Tables 4-12. Observing the results, the proposed method provides the most accurate overall estimates with the lowest mean square error. Furthermore, since ground truth measurements can be significantly biased [15]; the performance of each method was assessed by computing the peak signal-tonoise ratio (PSNR) of the motion compensated prediction error. Figure 13 shows the obtained results for each method and all the image pairs. The proposed scheme achieves marginally the best registration accuracy in comparison with $N G C$ [23], while the difference with the other methods is higher.

Additionally, the five MR images were used to evaluate the performance of each method in the presence of additive white Gaussian noise. In this case we 
Table 12: Average MSE with the corresponding PSNR values, and the estimated motion vectors for the 10 image pairs of the MRI data (Part 3).

\begin{tabular}{c|ccc|c|}
\hline \hline Image pairs & {$[3,5]$} & {$[4,5]$} & Average MSE $(\mathrm{x}, \mathrm{y})$ & PSNR dB \\
\hline GT & $(-2.40,4.00)$ & $(0.00,7.68)$ & $(0.0000,0.0000) \Rightarrow 0.0000$ & 0.0000 \\
Hoge & $(-2.18,4.51)$ & $(0.01,7.85)$ & $(0.3667,0.1914) \Rightarrow 0.5581$ & 30.2380 \\
Foroosh & $(-2.41,3.76)$ & $(-0.18,7.61)$ & $(0.3368,0.1945) \Rightarrow 0.5313$ & 30.3865 \\
Balci & $(-2.49,4.07)$ & $(-0.03,7.66)$ & $(0.5857,0.0841) \Rightarrow 0.6697$ & 30.0364 \\
Gaussian & $(-2.44,4.00)$ & $(-0.01,7.64)$ & $(0.3558,0.0324) \Rightarrow 0.3882$ & 30.7528 \\
Quadratic & $\mathbf{( - 2 . 2 7 , 4 . 0 0 )}$ & $(-0.01,7.78)$ & $(0.3334,0.0602) \Rightarrow 0.3936$ & 30.6963 \\
Sinc & $(-2.27,4.00)$ & $(0.00,7.87)$ & $(0.3490,0.1281) \Rightarrow 0.4771$ & 30.5317 \\
ESinc & $(-2.47,4.00)$ & $(0.00,7.54)$ & $(0.3834,0.0494) \Rightarrow 0.4329$ & 30.7081 \\
Ren & $(-2.41,4.00)$ & $(-0.02,7.64)$ & $(0.3488,0.0403) \Rightarrow 0.3892$ & 30.7583 \\
GC & $(-2.32,4.02)$ & $(-0.01,7.73)$ & $(0.3367,0.0297) \Rightarrow 0.3664$ & 30.7835 \\
NGC & $(-2.32,4.02)$ & $(-0.01,7.73)$ & $(0.3366,0.0299) \Rightarrow 0.3664$ & 30.7835 \\
Xiaohua & $(-2.39,4.04)$ & $\mathbf{( - 0 . 0 2 , 7 . 7 0 )}$ & $(0.3399,0.0411) \Rightarrow 0.3810$ & 30.7700 \\
HOGPC & $(-2.35,4.04)$ & $(0.01,7.72)$ & $(0.3301,0.0301) \Rightarrow \mathbf{0 . 3 6 0 1}$ & $\mathbf{3 0 . 7 9 0 1}$ \\
\hline
\end{tabular}

assume that the correct shift is given by the corresponding noise-free estimate for each method and image pair. In figure 14 the mean value of the registration error for noise variance in the range $[0.005,0.045]$ is illustrated for each method. Observing the results it can be seen that the proposed method is one of the most stable at high noise variances and provides the lowest overall MSE error. In the case of the other methods, the error rapidly increases for noise beyond a certain level, since they do not always provide the correct pixel accuracy. The proposed $H O G P C$ scheme exploiting the accuracy of HOG over noisy data allows precise estimates even for noise variance over the above range. Also, the PSNR was used to further compare the proposed scheme with the other state-of-the-art methods in the case of noise and the obtained results are shown in figure 15 demonstrating further the accuracy of $H O G P C$ in terms of motion compensated prediction error. Furthermore, experiments were performed with 8 different levels of motion blur. In each case the variance was increased moving from 0.25 up to 2 and for each level five repetitions were performed. The overall results 
Table 13: Average MSE of the estimated motion vectors with the corresponding PSNR values, for the 10 image pairs of the MRI data using 8 different motion blur levels and 5 repetitions for each one.

\begin{tabular}{c|ccccccc}
\hline \hline Method & GC & NGC & HOGPC & Hoge & Foroosh & Xiaohua & Ren \\
\hline PSNR & 39.4322 & 39.4368 & $\mathbf{3 9 . 4 3 8 4}$ & 36.7849 & 38.5084 & 39.4287 & 39.3615 \\
MSE & 0.0220 & 0.0117 & 0.0114 & 0.3692 & 0.0183 & 0.0102 & $\mathbf{0 . 0 0 7 9}$ \\
\hline
\end{tabular}

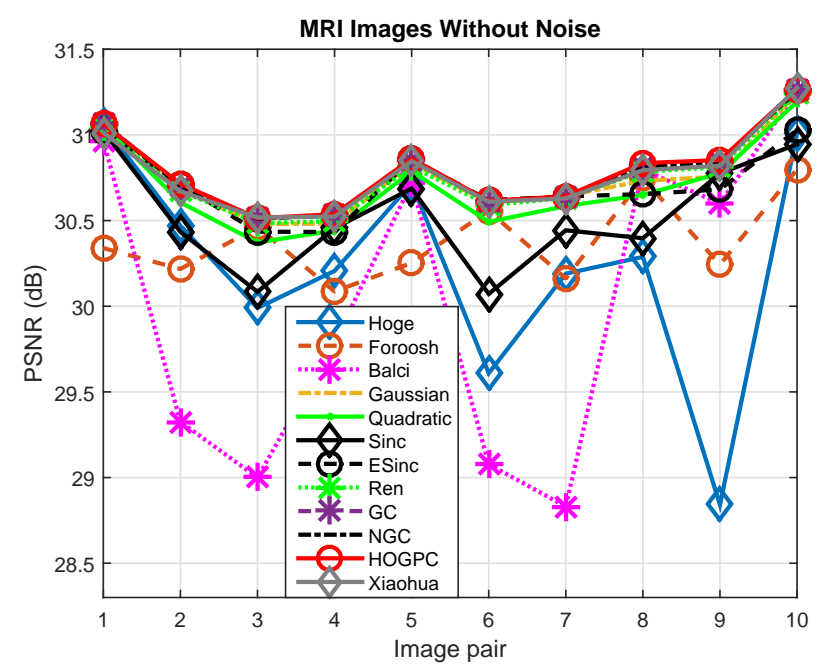

Figure 13: The PSNR values in $\mathrm{dB}$ over all image pairs.

are in Table 13 showing that most of the methods to have similar performance with the one in [22] and the proposed HOG-PC to result the best performance. The main advantage of the method in [22] is that very large blocks $256 \times 256$ are used in these experiments based on the MR data with ground truth. The proposed method outperforms significantly the other methods mainly in cases of small blocks e.g. $8 \times 8$ which are commonly used in the case of the video sequences and demonstrated in the previous section.

Overall the complexity of the proposed HOG-PC is higher compared to most of the other approaches due to the computational power required for the preprocessing stage and the estimation of the dense HOG transform. In this work all the methods were implemented in Matlab and the average required time per 


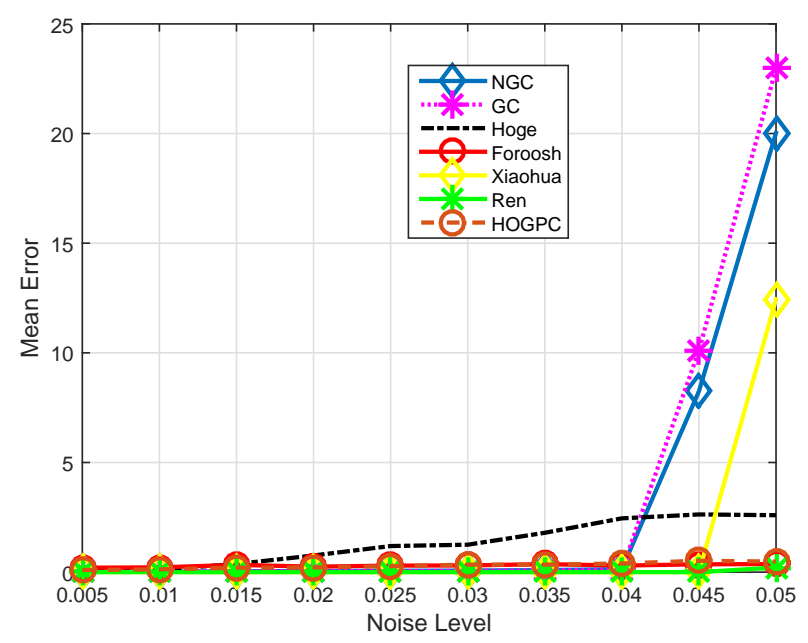

Figure 14: The Mean Error over all image pairs with different levels of noise for the top five methods.

Table 14: Average complexity for each method per frame over all the video sequences.

\begin{tabular}{c|cccccccc}
\hline \hline Method & GC & NGC & HOGPC & Hoge & Foroosh & Xiaohua & PC & Ren \\
\hline Time $(\mathrm{sec})$ & 0.3824 & 0.4340 & 0.6988 & 0.1826 & 0.0701 & 1.5374 & 0.0372 & 0.0389 \\
\hline
\end{tabular}

method is shown in Table 14. In the current architecture we did not considered any parallel implementations, but if a GPU-HOG transform [25] was used it could be no significant difference among them.

\section{Conclusion}

In this paper, a phase correlation technique based on histograms of oriented gradients that operates in the frequency domain for subpixel image registration was presented. The proposed method takes full account of all the advantages of HOG filter providing especially higher accuracy in small block sizes. One of the most attractive features of the proposed scheme is that it retains the orientation information and the corresponding weights of HOG filter and exploits its robustness to noise. HOG phase correlation yields very accurate subpixel 


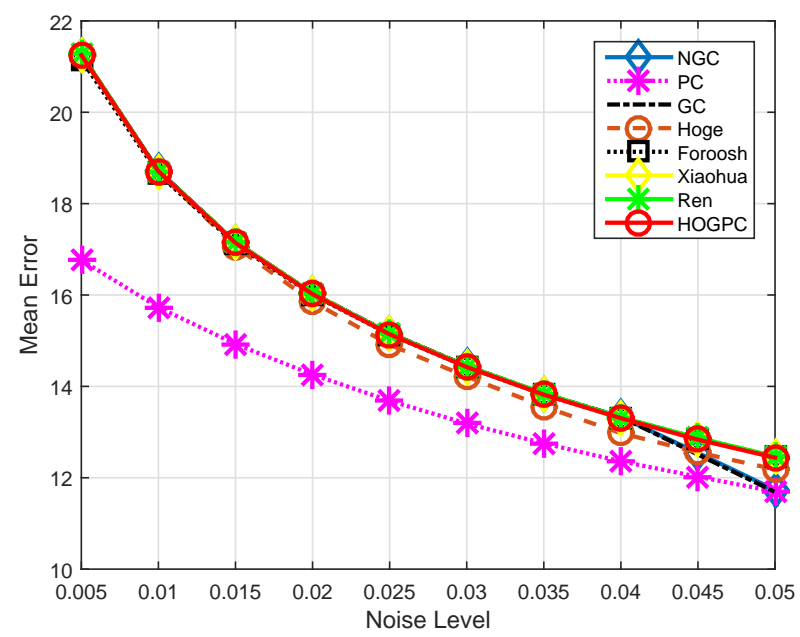

Figure 15: The PSNR values in $\mathrm{dB}$ over all image pairs with different levels of noise.

motion estimates for a variety of test material and motion scenarios and outperforms techniques, which are the current registration methods of choice in the frequency domain.

[1] C. Kuglin, D. Hines, The phase correlation image alignment method, in: Proc. IEEE Conf. Cyber. and Soc., 1975, pp. 163-165.

[2] J. Pearson, D. Hines, S. Goldsman, C. Kuglin, Video rate image correlation processor, Proc. SPIE Application of Digital Image Processing 119.

[3] G. Thomas, Television motion measurement for datv and other applications, BBC Res. Dept. Rep., No. 1987/11.

[4] B. Girod, Motion-compensating prediction with fractionalpel accuracy, IEEE Trans. Comm. 41 (4) (1993) 604.

[5] N. Dalal, B. Triggs, Histograms of oriented gradients for human detection, in: Proceedings of the 2005 IEEE Computer Society Conference on Computer Vision and Pattern Recognition (CVPR'05) - Volume 1 - Volume 01, CVPR '05, 2005, pp. 886-893. 
[6] I. Abdou, Practical approach to the registration of multiple frames of video images, in Proc. SPIE Conf. Vis. Commun. Image Process. 3653 (1999) $371-382$.

[7] V. Argyriou, T. Vlachos, A study of sub-pixel motion estimation using phase correlation, in Proc. Brit. Mach. Vis. Assoc. (2006) 387-396.

[8] S. Kruger, A. Calway, A multiresolution frequency domain method for estimating affine motion parameters, In Proc. IEEE International Conf. on Image Processing (1996) 113116.

[9] X. Tong, Z. Ye, Y. Xu, S. Liu, L. Li, H. Xie, T. Li, A novel subpixel phase correlation method using singular value decomposition and unified random sample consensus, Geoscience and Remote Sensing, IEEE Transactions on 53 (8) (2015) 4143-4156. doi:10.1109/TGRS.2015.2391999.

[10] L. Zhongke, Y. Xiaohui, W. Lenan, Image registration based on hough transform and phase correlation, Neural Networks and Signal Processing, 2003. Proceedings of the 2003 International Conference on 2 (2003) 956959.

[11] V. Maik, E. Chae, L. Eunsung, P. Chanyong, J. Gwanghyun, P. Sunhee, H. JinHee, J. Paik, Robust sub-pixel image registration based on combination of local phase correlation and feature analysis, Consumer Electronics (ISCE 2014), The 18th IEEE International Symposium on (2014) 1-2.

[12] M. Uss, B. Vozel, V. Dushepa, V. Komjak, K. Chehdi, A precise lower bound on image subpixel registration accuracy, Geoscience and Remote Sensing, IEEE Transactions on 52 (6) (2014) 3333-3345.

[13] P. Cheng, C.-H. Menq, Real-time continuous image registration enabling ultraprecise 2-d motion tracking, Image Processing, IEEE Transactions on 22 (5) (2013) 2081-2090.

[14] X. Tong, Y. Xu, Z. Ye, S. Liu, L. Li, H. Xie, F. Wang, S. Gao, U. Stilla, An improved phase correlation method based on 2-d plane fitting and the 
maximum kernel density estimator, Geoscience and Remote Sensing Letters, IEEE 12 (9) (2015) 1953-1957.

[15] W. Hoge, Subspace identification extension to the phase correlation method, IEEE Trans. Med. Imag. 22 (2) (2003) 277280.

[16] Y. Keller, A. Averbuch, A projection-based extension to phase correlation image alignment, Signal Process. 87 (2007) 124-133.

[17] M. Balci, H. Foroosh, Subpixel estimation of shifts directly in the fourier domain, IEEE Trans. Image Process. 15 (7) (2006) 1965-1972.

[18] H. Stone, M. Orchard, E. Chang, S. Martucci, A fast direct fourier-based algorithm for subpixel registration of images, IEEE Trans. Geosci. Remote Sens. 39 (10) (2001) 2235-2243.

[19] P. Vandewalle, S. Susstrunk, M. Vetterli, A frequency domain approach to registration of aliased images with application to superresolution, EURASIP J. Appl. Signal Process. (2006) 1-14.

[20] H. Foroosh, J. Zerubia, M. Berthod, Extension of phase correlation to subpixel registration, IEEE Trans. Image Process. 11 (2) (2002) 188-200.

[21] J. Ren, T. Vlachos, J. Jiang, Subspace extension to phase correlation approach for fast image registration, in Proc. IEEE ICIP (2007) 481-484.

[22] J. Ren, J. Jiang, T. Vlachos, High-accuracy sub-pixel motion estimation from noisy images in fourier domain, Image Processing, IEEE Transactions on 19 (5) (2010) 1379-1384.

[23] G. Tzimiropoulos, V. Argyriou, T. Stathaki, Subpixel registration with gradient correlation, Image Processing, IEEE Transactions on 20 (6) (2011) $1761-1767$.

[24] S. Mallat, A wavelet tour of signal processing, 2nd ed. New York: Academic.

[25] V. Prisacariu, I. Reid, Fasthog - a real-time gpu implementation of hog, Department of Engineering Science, Oxford University 09 (2310). 